\title{
Quantification of the Retinal Vascular Network Structure: Towards Effective Monitoring of Vessel Architecture Modulation in Diabetic Retinopathy
}

\author{
Delia Cabrera Debuc ${ }^{1}$ and William E. Smiddy ${ }^{1}$ \\ Bascom Palmer Eye Institute, University of Miami, Miami, FL, USA \\ \{dcabrera2@med.miami.edu, wsmiddy@med.miami.edu\}
}

\begin{abstract}
Diabetic retinopathy is the most common diabetic eye disease leading to vision loss. Disturbances have been detected in many aspects of ocular circulation in diabetes. However, hemodynamic and vessel architectural modulation have not been extensively investigated in DR. Therefore, an objective test for the early diagnosis, progression and therapeutic evaluation of DR based on vascular remodeling may aid to identify the individuals at great risk for visionthreatening problems. In this paper, we present a methodology to characterize the microvascular network structure in diabetic individuals. Our preliminary results may pave the path towards effective monitoring of vessel architecture modulation in diabetic retinopathy.
\end{abstract}

Keywords: diabetic retinopathy, microvascular network, vascular remodeling, and capillary perfusion map

\section{Introduction.}

Diabetes mellitus, a lifelong progressive disease, is the result of body's inability to produce insulin or use insulin to its full potential, and is characterized by high circulating glucose. This disease has reached epidemic proportion and has become one of the most challenging health problems of the 21st century. [1] Everyone with diabetes mellitus is at risk of developing diabetic retinopathy (DR), which is a wellcharacterized, sight-threatening microvascular complication leading to vision loss in millions of patients in industrialized and developed countries. [2] In spite of extensive research, DR has remained difficult to prevent and treat. The main ocular target of DR is the retina that develops intravitreal hemorrhages, macular edema, retinal detachments and neovascularization. However, $70 \%$ of diabetic patients also suffer from corneal abnormalities. The pathogenesis of DR is complex and still remains incompletely understood. Although several biochemical mechanisms, most notably hyperglycemia, have been proposed as possible pathogenic factors in DR, there remains 
controversy regarding the initiating events. It has been postulated that retinal vasodilation and retinal hyperperfusion, possibly related to hypoxia and the release of nitric oxide, may be key initiating factors in early DR developments. [3] Hyperperfusion, in turn, has been associated with impaired vascular autoregulation, which then may lead to an increase in epithelial wall tension and permeability, and subsequent leakage and microaneurysm development. [4] Disturbances have been detected in many aspects of ocular circulation in diabetes. However, hemodynamic and vessel architectural's modulation have not been extensively investigated in DR. Therefore, an objective test for the early diagnosis, progression and therapeutic evaluation of DR based on vascular remodeling may aid to identify the individuals at great risk for vision-threatening problems.

Methods currently used in eye screening examinations have their own unique drawbacks. Currently, available offerings are generally based on dilated examinations and fundus photography analysis. Dilated examinations reveal the entire retina, but you must record the results by drawing a color diagram in the patient's chart. Fundus cameras produce a more objective, permanent record of retinal morphology, but they can be difficult to use and capture only a small portion of the central fundus. By evaluating the fundus of diabetic children and adolescents using fundus photography, Falck and Laatikainen reported that the blood vessel diameter significantly increased with the increase in the duration of DR. [5] They also found that signs of early retinopathy developed more often in eyes with venous dilatation than in those without. In addition, retinal vasodilation has been proposed to occur before the onset of clinically evident DR. Therefore, there is a need to reveal the precise nature of the disturbance of retinal hemodynamic in a defined population of patients with diabetes using better techniques and methods.

The method was based on large retinal vessels visible on color fundus photographs. The ability of imaging the microvascular capillary network may provide crucial information on the onset and progression of other diabetic complications. [6] Subclinical retinal microvascular abnormalities such as marked capillary contortions and dilatations of venous branches were observed in T1DM. [7-9] Using a scanning laser technique on the retinal fluorescein angiogram, the reduction in capillary blood flow velocities and enlargement of perifoveal inter-capillary areas and foveal avascular zone were clear. [9] This method requires the injection of a fluorescein dye, which represents a limitation in routine pediatric practice of T1DM patients. Recent advances in optical imaging techniques enable imaging the retinal microvasculature at the capillary level and generate high-resolution non-invasive capillary perfusion maps (nCPMs) using a commercially available Retinal Function Imager (RFI, Optical Imaging Ltd, Rehovot, Israel). The RFI is a novel device that is designed to noninvasively and directly measure microcirculation of the retinal small vessels and quantify retinal small vessel morphology in great details. [10] The RFI is the first available U.S. Food and Drug Administration (FDA) approved retinal function imaging device, which performs retinal angiograms without any contrast agent injection such as fluorescein. 
As it can be seen, there is a need for knowing the natural history of structural alterations, hemodynamic disturbances and functional loss in DR, which can greatly aid in developing strategies to detect both early DR and eventual DR progression. In this paper, we present a methodology to characterize the microvascular network structure in diabetic individuals. Our preliminary results may pave the path towards effective monitoring of vessel architecture modulation in diabetic retinopathy.

\section{Methods}

\subsection{Collection of data}

The study was approved by the Institutional Review Board of the University of Miami, Miami, FL, USA. The research adhered to the tenets set forth in the declaration of Helsinki and written informed consent was obtained from the subjects enrolled in this cross-sectional study. A total of 15 eligible eyes from 13 participants were analyzed, which included a total of 5 healthy eyes ( $32 \pm 6$ years, 2 female, 3 male), 5 diabetic eyes with no retinopathy (DM, $31 \pm 10$ years, 1 female, 4 male) and 5 eyes with mild diabetic retinopathy (MDR, $45 \pm 16$ years, 4 female, 1 male).

\subsection{Microvascular Imaging}

The RFI device has been described in details elsewhere [10-14] and its measurement variability has been validated. $[15,16]$ RFI is a commercially available, fundus camera based, image modality that captures reflectance changes as a function of time under stroboscopic illumination (wavelengths between 530 and $590 \mathrm{~nm}$ ). It is designed to directly and non-invasively measure the retinal vessel flow velocity and to perform capillary perfusion mapping in great details. [17] Using a fundus camera based photography, the retina vessel tree can be tracked up to 3rd branching for vessel network analysis (e.g. density and morphology, see Fig. 1A) and the RFI can image up to 6th branching, [18] as demonstrated in our Fig. 1B. In terms of blood flow velocity calculations, RFI can measure blood flow velocity reliably up to 3rd branching of the retinal vessels. 

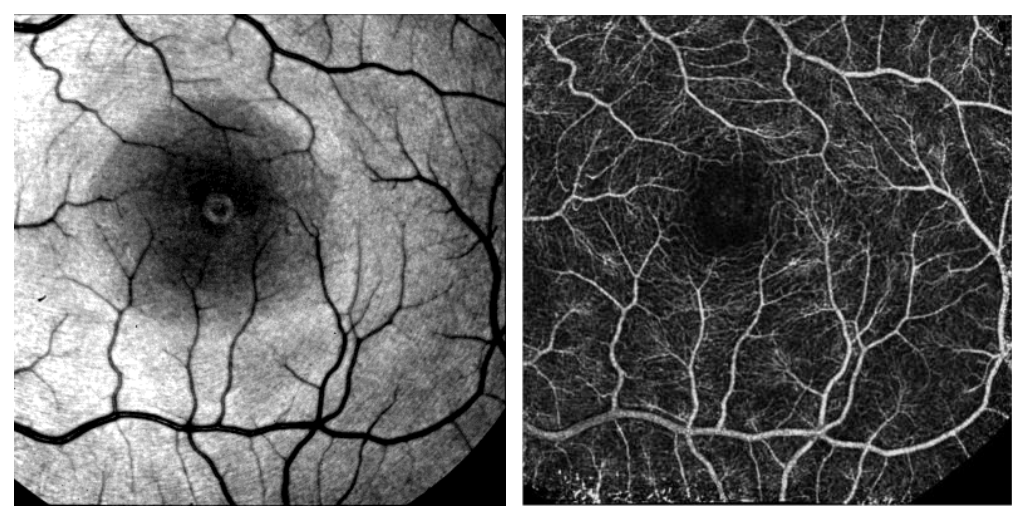

Fig. 1. Human retinal microvascular network of a healthy subject assessed by the RFI. The fovea (left) was imaged and capillary perfusion (right) was obtained.

\subsection{Microvascular modulation parameters}

Complexity index (CI): A Fourier fractal dimension (FFD) approach was used to compute the fractal dimension of the RFI's nCPM images (grey level images). The advantage of the FFD approach is that it computes the fractal dimension and eliminates the need for image segmentation. [19]

Vascular coverage (VC): RFI's nCPM images (see Fig. 1B) were skeletonized (1 pixel-wide segments) to determine the centerline of each vessel. Then, the skeleton was superposed on the binary image obtained from the original RFI's image (see Fig. 1A) to locate the boundary of each vessel and calculate the extent or radius at each pixel orthogonal to the direction of the centerline of the vessel. A histogram of the radii' $\mathrm{s}$ distribution was constructed after creating an array of radii for which each radius is assigned into a 2-pixel wide pack.

\subsection{Statistical analysis}

In order to test for statistically significant differences, paired standard Student $t$ tests were used. Statistical significance was asserted at a p-value $<0.05$

\section{$3 \quad$ Results}

Differences in VC were observed in diabetic patients when compared to normal healthy subjects. Particularly, the blood vessel radius distribution analysis showed a change in vessel radius (see Fig. 2) when comparing MDR with DM (starting at [4,6] pixel range) and normal healthy eyes (starting at the $[6,8]$ pixel range). The distribution trend, although showed proportional VC without significant differences between diabetic and healthy eyes, revealed that a relatively larger amount of diabetic blood vessels occupies larger ranges ([6 to 16 pixels]). No significant differences $(p>0.05)$ 
were observed for the CI when comparing healthy eyes with diabetic eyes with and without early retinopathy. The CI was $1.65 \pm 0.02$ and $1.67 \pm 0.01$ in MDR and healthy eyes, respectively. Figure 3 shows the capillary perfusion map obtained for a diabetic subject with MDR.

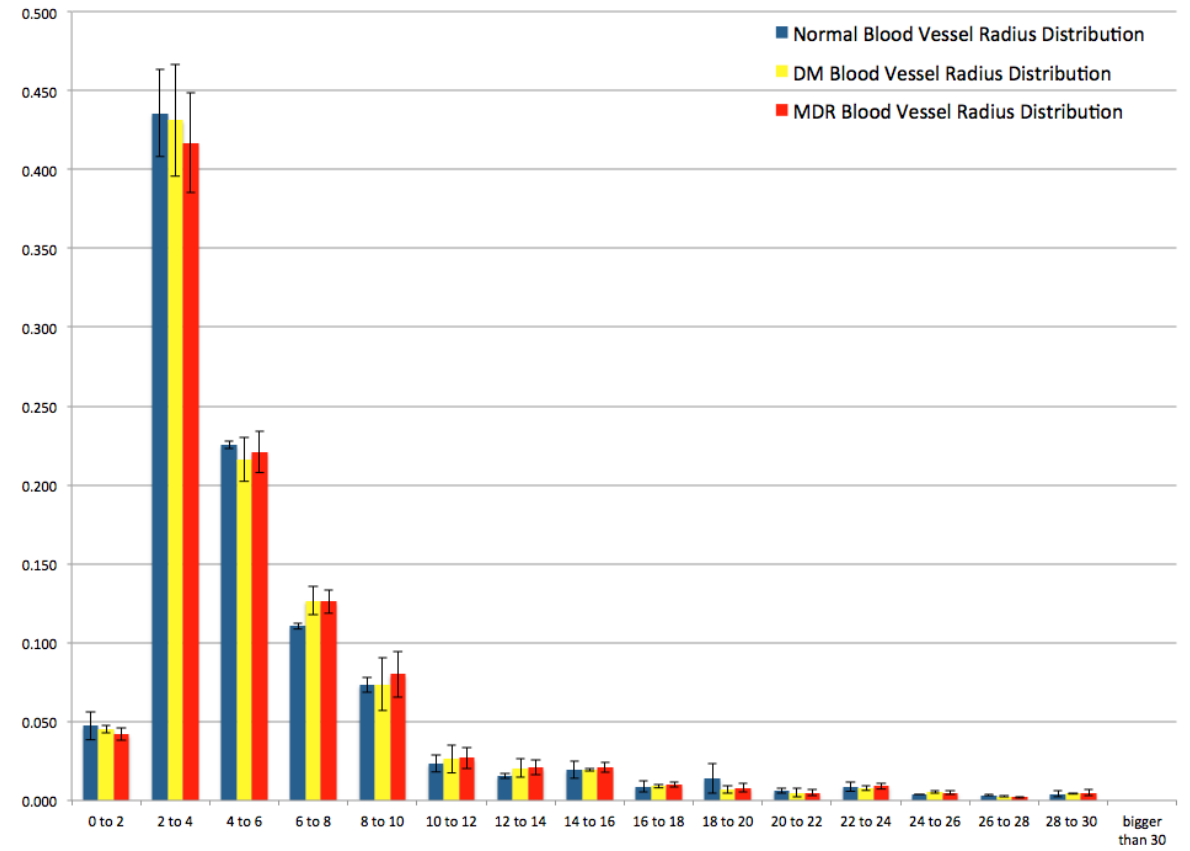

Fig. 2. Histogram of mean radius distribution across a wide range of pixel radius ranges [Proportion (y axis) vs. radius in pixels ( $\mathrm{x}$ axis)].

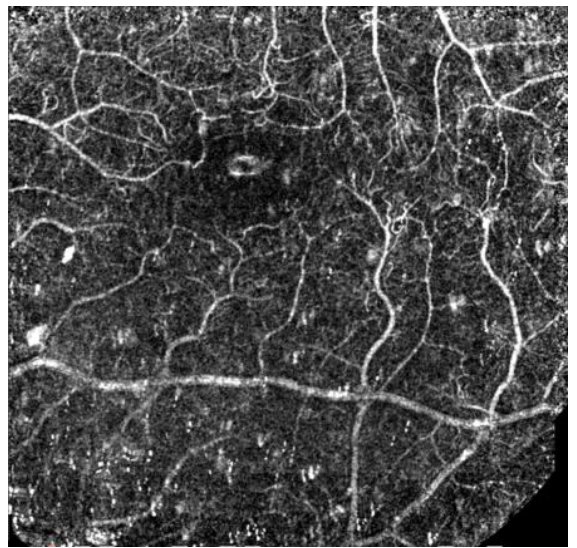

Fig. 3. Capillary perfusion maps obtained for a diabetic subject with MDR 


\section{Discussion and Conclusions}

Extensive research has been conducted to determine effective methods of detection and treatment of diabetes mellitus and its attendant ocular complications. Studying methods to detect these complications in their earliest stages may inspire development of early treatment strategies before clinical signs are apparent, mitigating irreversible damage to the microvasculature. Results of our small cross-sectional study demonstrate the feasibility of optical imaging using the RFI for obtaining high-resolution nCPMs and providing the characteristics of the retinal vessels in diabetic patients as well as their remodeling features as result of the disease progression or in response to treatment. Our study also suggest that a larger longitudinal study would corroborate more convincingly whether larger proportion of diabetic blood vessels could dominate larger pixel radius ranges as DR progress. In summary, this study demonstrates a possible role for RFI as an in vivo platform to monitor vessel architecture modulation in DR and may also allow new insights on the pathogenesis of vascular changes in the diabetic eye.

Acknowledgments. This study was supported in part by the research grants NIH R01EY020607, NIH R01EY020607S, NIH Center Grant P30 EY014801 and the grant from Research to Prevent Blindness (RPB).

\section{$5 \quad$ References}

1. Frank, R.N.: Diabetic retinopathy, New England Journal of Medicine, vol. 350, no. 1, pp. 48-58, (2004)

2. Hoowerf, B.: Complications of diabetes mellitus. Int. J. Diab. Dev. Countries, vol. 25, pp. 63-69 (2005)

3. Klein R, Klein BE. : Diabetic eye disease. Lancet; 350:197-204. (1997)

4. Klein R, Klein BE. : Vision disorders in diabetes. In: National diabetes data group, ed. Diabetes in America, $2^{\text {nd }}$ edn. Washington, DC: US Government printing office: 293-338. (1995)

5. Falck A, Laatikainen L.: Retinal vasodilation and hyperglycaemia in diabetic children and adolescents. Acta Ophthalmol Scand; 73:119-24. (1995)

6. Tam J, Dhamdhere KP, Tiruveedhula P, et al.: Subclinical capillary changes in nonproliferative diabetic retinopathy. Optom Vis Sci. 89:E692-E703. (2012)

7. Koscielny J, Latza R, Wolf S, Kiesewetter H, Jung F. : Early rheological and microcirculatory changes in children with type I diabetes mellitus. Clin Hemorheol Microcirc.;19:139-150. (1998)

8. Arend $\mathrm{O}$, Wolf $\mathrm{S}$, Jung $\mathrm{F}$, et al. : Retinal microcirculation in patients with diabetes mellitus: dynamic and morphological analysis of perifoveal capillary network. $\mathrm{Br} \mathrm{J}$ Ophthalmol.;75:514-518. (1991)

9. Izhaky D, Nelson DA, Burgansky-Eliash Z, Grinvald A. : Functional imaging using the retinal function imager: direct imaging of blood velocity, achieving fluorescein angiography-like images without any contrast agent, qualitative oximetry, and functional metabolic signals. Jpn J Ophthalmol. ;53:345-351. (2009) 
10. Beutelspacher SC, Serbecic N, Barash H, et al. : Retinal blood flow velocity measured by retinal function imaging in retinitis pigmentosa. Graefes Arch Clin Exp Ophthalmol.; 249:1855-1858. (2011)

11. Landa G, Jangi AA, Garcia PM, Rosen RB. : Initial report of quantification of retinal blood flow velocity in normal human subjects using the Retinal Functional Imager (RFI). Int Ophthalmol.; 32:211-215. (2012)

12. Nelson DA, Krupsky S, Pollack A, et al. : Special report: Noninvasive multi-parameter functional optical imaging of the eye. Ophthalmic Surg Lasers Imaging.;36:57-66. (2005)

13. Landa G, Rosen RB. : New patterns of retinal collateral circulation are exposed by a retinal functional imager (RFI). Br J Ophthalmol.;94:54-58. (2010)

14. Nelson DA, Burgansky-Eliash Z, Barash H, et al. : High-resolution wide-field imaging of perfused capillaries without the use of contrast agent. Clin Ophthalmol. ;5:1095-1106. (2011)

15. Chhablani J, Bartsch DU, Cheng L, et al. : Segmental reproducibility of retinal blood flow velocity measurements using retinal function imager. Graefes Arch Clin Exp Ophthalmol. (2013).

16. Burgansky-Eliash Z, Lowenstein A, Neuderfer M, et al. : The correlation between retinal blood flow velocity measured by the retinal function imager and various physiological parameters. Ophthalmic Surg Lasers Imaging Retina.; 44:51-58. (2013)

17. Izhaky D, Nelson DA, Burgansky-Eliash Z, Grinvald A. : Functional imaging using the retinal function imager: direct imaging of blood velocity, achieving fluorescein angiography-like images without any contrast agent, qualitative oximetry, and functional metabolic signals. Jpn J Ophthalmol.; 53:345-351. (2009)

18. Witkin AJ, Alshareef RA, Rezeq SS, et al.: Comparative Analysis of the Retinal Microvasculature Visualized With Fluorescein Angiography and the Retinal Function Imager. Am J Ophthalmol. (2012).

19. Azemin MZC, Kumar DK, Wong TY, Wang JJ, Kawasaki R, et al.: Fusion of multiscale wavelet-based fractal analysis on retina image for stroke prediction. Engineering in Medicine and Biology Society (EMBC), 2010 Annual International Conference of the IEEE: 4308-4311. doi: 10.1109/iembs.2010.5626209, (2010) 